\title{
THE $k$-FUNCTION, A PARTICULAR CASE OF THE CONFLUENT HYPERGEOMETRIC FUNCTION*
}

BY

\section{H. BATEMAN}

In his well known paper $†$ in which he defines the function $W_{k, m}(z)$ Professor E. T. Whittaker says: "There are other members of the family of functions $W_{k, m}(z)$ which have not hitherto been noticed, but which give promise of interesting properties. Among these may be mentioned the families of functions for which $m=0$ and those for which $m=\frac{1}{2}$." The functions considered here correspond to the case $m=\frac{1}{2}$. The associated differential equation has arisen recently in the theory of turbulence, particularly in the researches of W. Tollmienł and Th. von Kármán.

1. Definition of the functions. The function $k_{n}(x)$ may be defined for real values of $x$ and $n$ by the definite integral

$$
k_{n}(x)=\frac{2}{\pi} \int_{0}^{\pi / 2} \cos (x \tan \theta-n \theta) d \theta .
$$

When $n=0$ we have an integral which is easily evaluated, in fact

$$
k_{0}(x)=e^{-|x|} .
$$

For other values of $n$ we have the interesting relations

$$
\begin{aligned}
k_{-n}(x) & =k_{n}(-x), \\
k_{n}(0) & =\frac{2}{n \pi} \sin \frac{n \pi}{2},
\end{aligned}
$$

which follow immediately from the definition. When $n$ is an integer the definite integral may be evaluated in terms of known functions. In particular

$$
k_{2}(x)=(x+|x|) e^{-|x|} .
$$

This expression shows that $k_{2}(x)$ is zero when $x$ is negative and it will be seen presently that if $n$ is a positive integer $k_{2 n}(x)$ is also zero when $x$ is negative.

* Presented to the Society, September 9, 1931; received by the editors November 10, 1930. The $k$-notation has been adopted in honor of Dr. Th. von Kármán, who submitted the differential equation to the present author for investigation.

$\dagger$ Bulletin of the American Mathematical Society, vol. 10 (1903-04), p. 133.

$\ddagger$ Göttinger Nachrichten, 1929, p. 21. Put $U=c+b y$ in equation (2). [Noted by C. B. Millikan.]

$\$$ International Congress of Applied Mechanics, Stockholm, 1930. Göttinger Nachrichten, 1930, p. 58. Put $\psi=\alpha e^{h x} Y(y)$ in equation (8) and neglect $\alpha^{2}$. 
Since $|\cos (x \tan \theta-n \theta)| \leqq 1$, we have the important inequality

$$
\left|k_{n}(x)\right| \leqq 1,
$$

which holds for all real values of $n$ and $x$. When $n$ is an odd integer the function $k_{n}(x)$ may be expressed in terms of the Bessel functions $K_{0}(x)$ and $K_{1}(x)$. In particular, if $t=\tan \theta$,

$$
\begin{aligned}
k_{1}(x) & =\frac{2}{\pi} \int_{0}^{\infty} \frac{\cos (x t) d t}{\left(1+t^{2}\right)^{3 / 2}}+\frac{2}{\pi} \int_{0}^{\infty} \frac{\sin (x t) t d t}{\left(1+t^{2}\right)^{3 / 2}} \\
& =\frac{2}{\pi} \int_{0}^{\infty} \frac{\cos (x t) d t}{\left(1+t^{2}\right)^{3 / 2}}-\frac{2 x}{\pi} \int_{0}^{\infty} \frac{\cos (x t) d t}{\left(1+t^{2}\right)^{1 / 2}} \\
& =\frac{2 x}{\pi}\left[K_{1}(x)-K_{0}(x)\right], x>0, \\
& =-\frac{2 x}{\pi}\left[K_{1}(-x)+K_{0}(-x)\right], x<0 .
\end{aligned}
$$

2. The generating function. When $n$ is an even integer the function $k_{n}(x)$ may be defined with the aid of the expansion

$$
\begin{gathered}
e^{i x \tan (\theta+i \alpha)=k_{0}(x)+}+k_{2}(x) e^{2 i \theta-2 \alpha}+k_{4}(x) e^{4 i \theta-4 \alpha}+\cdots, \\
\alpha>0, \quad x>0 .
\end{gathered}
$$

If $s=e^{2 i \theta-2 \alpha}$, the expansion becomes

$$
e^{-x(1-s) /(1+s)}=k_{0}(x)+s k_{2}(x)+s^{2} k_{4}(x)+\cdots,
$$

and it is readily seen that the power series in $s$ is absolutely convergent when $|s|<1$. The convergence on the circle of convergence will be studied later; it will suffice now to say that the series takes the form

$$
e^{i x \tan \theta}=k_{0}(x)+k_{2}(x) e^{2 i \theta}+k_{4}(x) e^{4 i \theta}+\cdots,
$$

and is the Fourier series of the function $e^{i x \tan \theta}$ for the interval $(-\pi<\theta<\pi)$. Indeed,

$$
\begin{aligned}
\frac{1}{2 \pi} \int_{-\pi}^{\pi} e^{i x \tan \theta-2 n i \theta} d \theta & =\frac{1}{\pi} \int_{0}^{\pi} \cos [x \tan \theta-2 n \theta] d \theta \\
& =\frac{2}{\pi} \int_{0}^{\pi / 2} \cos [x \tan \theta-2 n \theta] d \theta=k_{2 n}(x) .
\end{aligned}
$$

The fact that $k_{2 n}(x)=0$ when $n$ is a negative integer accounts for the absence of terms of type $k_{-2 m}(x)$ and enables us to write the expansion in the usual form, 


$$
e^{i x \tan \theta}=\sum_{m=-\infty}^{\infty} k_{2 m}(x) e^{2 m i \theta} .
$$

The expansion (2.2) shows that the function $k_{2 m}(x)$ is closely related to the generalized polynomial of Laguerre which is defined by Sonine's expansion*

$$
(1-z)^{-1-\alpha} e^{-x z /(1-z)}=\sum_{n=0}^{\infty} L_{n}^{(\alpha)}(x) z^{n} .
$$

Indeed, if $L_{n}(x)$ denotes the polynomial of Lagrange and Laguerre, we have Abel's expansion

$$
(1-z)^{-1} e^{-x z /(1-z)}=\sum_{n=0}^{\infty} L_{n}(x) z^{n},
$$

and it is at once seen that when $x>0$,

$$
k_{2 m}(x)=(-1)^{m} e^{-x}\left[L_{m}(2 x)-L_{m-1}(2 x)\right] .
$$

This is a particular case of the more general formula

$$
\begin{aligned}
k_{2 m}(x)= & (-1)^{m} e^{-x}\left[L_{m}^{(\alpha)}(2 x)-\left(\begin{array}{c}
\alpha+1 \\
1
\end{array}\right) L_{m-1}^{(\alpha)}(2 x)\right. \\
& \left.+\left(\begin{array}{c}
\alpha+1 \\
2
\end{array}\right) L_{m-2}^{(\alpha)}(2 x)+\cdots+(-1)^{m}\left(\begin{array}{c}
\alpha+1 \\
m
\end{array}\right) L_{0}^{(\alpha)}(2 x)\right]
\end{aligned}
$$

which is proved by equating the coefficients of $z^{m}$ on the two sides of the equation

$$
e^{x}\left[k_{0}(x)-z k_{2}(x)+z^{2} k_{4}(x)-\cdots\right]=(1-z)^{\alpha+1} \sum_{n=0}^{\infty} z^{n} L_{n}^{(\alpha)}(2 x) .
$$

A reciprocal relation $\dagger$

$$
\begin{aligned}
& (-1)^{n} e^{-x} L_{n}^{(\alpha)}(2 x) \\
& \quad=k_{2 n}(x)-\left(\begin{array}{c}
\alpha+1 \\
1
\end{array}\right) k_{2 n-2}(x)+\left(\begin{array}{c}
\alpha+2 \\
2
\end{array}\right) k_{2 n-4}(x)-\cdots
\end{aligned}
$$

is obtained by equating the coefficients of $z^{n}$ on the two sides of the equation

$$
\sum_{n=0}^{\infty} e^{-x} L_{n}^{(\alpha)}(2 x) z^{n}=(1-z)^{-\alpha-1}\left[k_{0}(x)-z k_{2}(x)+z^{2} k_{4}(x)-\cdots\right] .
$$

* N. Sonine, Mathematische Annalen, vol. 16 (1880), p. 1. In Sonine's notation

$$
L_{n}^{\alpha}(x)=(-1)^{n} \Gamma(n+\alpha+1) T_{\alpha^{n}}(x) .
$$

The notation used here is the same as that used by Hille and Szegö.

† S. Namuri, Tôhoku Mathematical Journal, vol. 30 (1928-29), p. 58. 
An important property of the function $k_{2 m}(x)$ may be deduced directly from equation (2.7) with the aid of the orthogonal relation*

$$
\begin{aligned}
\int_{0}^{\infty} e^{-u} L_{m}(u) L_{n}(u) d u & =0, m \neq n, \\
& =1, m=n .
\end{aligned}
$$

We have in fact

$$
\begin{aligned}
\int_{0}^{\infty}\left[k_{2 m}(x)\right]^{2} d x=1, m & >0, \\
=\frac{1}{2}, m & =0 ; \\
\int_{0}^{\infty} k_{2 m}(x) k_{2 m+2 s}(x) d x & =0, s>1, \\
& =\frac{1}{2}, s=1 .
\end{aligned}
$$

A second generating function may be derived from the expansion $\dagger$

$$
e^{-u}(u x)^{-m / 2} I_{m}\left(2(u x)^{1 / 2}\right)=\sum_{n=0}^{\infty} \frac{(-u)^{n}}{\Gamma(m+n+1)} L_{n}^{(m)}(x)
$$

by putting $m=0$ and using (2.7). The result is

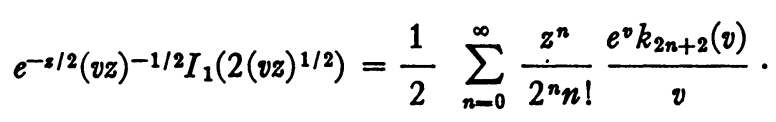

The function $I_{m}(y)$ is the Bessel function with imaginary argument.

3. The Lagrangian expansion. The expansion of the generating function may be derived by Lagrange's theorem from the implicit relation

$$
z=x-s z
$$

which may be used to define $z$ as a function of $s$. The expansion of the function

is then

$$
\frac{d z}{d x}\left(\frac{1}{z} e^{-2 z}\right)=\frac{1}{x} e^{-2 x /(1+8)}
$$

$$
\frac{1}{x} e^{-2 x /(1+8)}=\frac{1}{x} e^{-2 x}+\sum_{n=1}^{\infty}(-1)^{n} \frac{s^{n}}{n !} \frac{d^{n}}{d x^{n}}\left[e^{-2 x} x^{n-1}\right] .
$$

We thus have a representation of $k_{2 n}(x)$ for $x>0$,

$$
k_{2 n}(x)=\frac{(-1)^{n} x e^{x}}{n !} \frac{d^{n}}{d x^{n}}\left[e^{-2 x} x^{n-1}\right],
$$

which is analogous to Sonine's formula

* This relation was obtained by N. H. Abel, Oeuvres (Sylow and Lie), vol. II, p. 284.

$\dagger$ N. Sonine, loc. cit., p. 41. 


$$
L_{n}^{(\alpha)}(x)=\frac{1}{n !} e^{x} x^{-\alpha} \frac{d^{n}}{d x^{n}}\left[x^{n+\alpha} e^{-x}\right],
$$

for the generalized Laguerre polynomial.

4. The difference equations and inequalities. It is readily seen from (1.1) that

$$
\begin{aligned}
(n-2)\left[k_{n}(x)\right. & \left.+k_{n-2}(x)\right]+(n+2)\left[k_{n}(x)+k_{n+2}(x)\right]- \\
= & -\frac{8}{\pi} \int_{0}^{\pi / 2} \frac{d}{d \theta}\left[\cos ^{2} \theta \sin (x \tan \theta-n \theta)\right] d \theta \\
= & 0 .
\end{aligned}
$$

It is also seen that

$$
4 x k_{n}^{\prime}(x)=(n-2) k_{n-2}(x)-(n+2) k_{n+2}(x),
$$

for we have the relation

$$
\begin{aligned}
k_{n}^{\prime}(x)+k_{n+2}^{\prime}(x)= & -\frac{2}{\pi} \int_{0}^{\pi / 2}[\sin (x \tan \theta-n \theta) \\
& +\sin (x \tan \theta-n \theta-2 \theta)] \tan \theta d \theta \\
= & -\frac{4}{\pi} \int_{0}^{\pi / 2} \sin [x \tan \theta-n \theta-\theta] \sin \theta d \theta \\
= & +\frac{2}{\pi} \int_{0}^{\pi / 2}[\cos (x \tan \theta-n \theta)-\cos (x \tan \theta-n \theta-2 \theta)] d \theta,
\end{aligned}
$$

in which we must be careful to form the expression for $k_{n}(x)+k_{n+2}(x)$ as a definite integral before we differentiate to form an expression for the quantity on the left hand side. When the ensuing relation

$$
k_{n}^{\prime}(x)+k_{n+2}^{\prime}(x)=k_{n}(x)-k_{n+2}(x)
$$

is combined with (4.1) it leads to (4.2). It should be remarked that when the difference equation (4.1) is used to calculate $k_{n}(x)$ for even negative values of $n$, using the known values of $k_{n}(x)$ for positive even values of $\boldsymbol{n}$ it is found that when $x>0$ we have $k_{-2 m}(x)=0$ for all positive integral values of $m$. This is what was anticipated in $\$ 1$. With the aid of (1.5) and (4.1) we obtain the inequality

$$
\left|k_{n}(x)\right| \leqq \frac{|n|}{|x|} \quad(n>2) .
$$

Similarly (4.2) gives the inequality

$$
\left|k_{n}^{\prime}(x)\right| \leqq \frac{|n|}{2|x|} \quad(n>2) \text {. }
$$


These inequalities show that as $|x| \rightarrow \infty, k_{n}(x) \rightarrow 0$ and $k_{n}^{\prime}(x) \rightarrow 0$. Another useful inequality is obtained by combining (4.4) with (4.1):

$$
\left|k_{n}(x)\right|<\frac{n^{2}+2}{|x|^{2}} \quad(n>2) \text {. }
$$

When $s$ is a positive integer, $k_{2 s}(x)$ is zero for $x=0$ and is finite for positive values of $x$; consequently we can find a positive number $\phi(s)$ such that

$$
\left|k_{2 s}(x)\right| \leqq x \phi(s), 0 \leqq x \leqq 1
$$

This inequality will be used later in combination with (4.4) and (4.6).

5. The differential equation. The relation (4.2) gives that is,

$$
4 x k_{n}^{\prime \prime}(x)+4 k_{n}^{\prime}(x)=(n-2) k_{n-2}^{\prime}(x)-(n+2) k_{n+2}^{\prime}(x),
$$

$$
\begin{aligned}
4 x k_{n}^{\prime \prime}(x) & =(n-2)\left[k_{n-2}^{\prime}(x)+k_{n}^{\prime}(x)\right]-(n+2)\left[k_{n+2}^{\prime}(x)+k_{n}^{\prime}(x)\right] \\
& =(n-2)\left[k_{n-2}(x)-k_{n}(x)\right]-(n+2)\left[k_{n}(x)-k_{n+2}(x)\right] \\
& =4(x-n) k_{n}(x) .
\end{aligned}
$$

Hence the function $k_{n}(x)$ satisfies the differential equation

$$
x k_{n}^{\prime \prime}(x)=(x-n) k_{n}(x) .
$$

This is an equation of Laplace's type and is a degenerate form of the canonical equation adopted by Whittaker* in his study of the confluent hypergeometric functions.

6. The orthogonal relations. The usual method of deriving orthogonal relations from a differential equation suggests that we should consider the value of the definite integral

$$
I_{m, n}=\int_{-\infty}^{\infty} k_{2 m}(x) k_{2 n}(x) \frac{d x}{x}
$$

in which $m$ and $n$ are not simultaneously zero. When $m$ and $n$ are positive integers, a reduction formula

$$
\begin{aligned}
(n-1) I_{m, n-1}+(n+1) I_{m, n+1}+2 n I_{m, n} & =0, n>m+1, \\
& =1, n=m+1, \\
& =2, n=m, \\
& =1, n=m-1, \\
& =0, n<m-1,
\end{aligned}
$$

* Whittaker and Watson, Modern Analysis, chapter 16; see also H. A. Webb and J. R. Airey, Philosophical Magazine, (6), vol. 36 (1918), p. 129. 
for $I_{m, n}$ is readily derived from the difference equation (4.1) and the relations (2.91). When $n=1$ the formula (2.91) also gives

$$
\begin{aligned}
I_{m, 1} & =2 \int_{0}^{\infty} e^{-x} k_{2 m}(x) d x=0, m>1, \\
& =1, m=1,
\end{aligned}
$$

while (3.1) gives

$$
\begin{aligned}
I_{m, 0} & =\int_{0}^{\infty} e^{-x} k_{2 m}(x) \frac{d x}{x} \\
& =\frac{(-1)^{m}}{m !} \int_{0}^{\infty} \frac{d^{m}}{d x^{m}}\left[e^{-2 x} x^{m-1}\right] d x \\
& =(-1)^{m-1} \frac{1}{m}, m>0 .
\end{aligned}
$$

With the aid of these particular relations and the reduction formula the integral $I_{m, n}$ can be calculated step by step and is found to be zero when $m>1$ and $n<m$. On the other hand the reduction formula and the particular values give

$$
I_{m, m}=\frac{1}{m} \quad(m>0) .
$$

Hence, if $m>0$ the functions $k_{2 m}(x)$ form an orthogonal set. When $m$ and $n$ have any real values the integral $I_{m, n}$ may be understood to have its principal value. To find this we note that the differential equation gives

$$
\frac{d}{d x}\left[k_{2 n}(x) k_{2 m}^{\prime}(x)-k_{2 m}(x) k_{2 n}^{\prime}(x)\right]=\frac{2}{x}(n-m) k_{2 n}(x) k_{2 m}(x) .
$$

Therefore since $k_{2 n}{ }^{\prime}(x)$ and $k_{2 n}(x) \rightarrow 0$ as $|x| \rightarrow \infty$, we have

$$
\begin{aligned}
& \int_{\epsilon}^{\infty} k_{2 n}(x) k_{2 m}(x) \frac{d x}{x}=\frac{1}{2(m-n)}\left[k_{2 n}(\epsilon) k_{2 m}^{\prime}(\epsilon)-k_{2 m}(\epsilon) k_{2 n}^{\prime}(\epsilon)\right], \\
& \int_{-\infty}^{-\epsilon} k_{2 n}(x) k_{2 m}(x) \frac{d x}{x}=\frac{1}{2(m-n)}\left[k_{2 m}(-\epsilon) k_{2 n}^{\prime}(-\epsilon)-k_{2 n}(-\epsilon) k_{2 m}^{\prime}(-\epsilon)\right] .
\end{aligned}
$$

Now as $\epsilon \rightarrow 0$,

$$
k_{2 n}(\epsilon) \rightarrow k_{2 n}(-\epsilon)=\frac{1}{n \pi} \sin (n \pi),
$$

and the integral (1.1) gives 


$$
k_{2 n}^{\prime}(x)=-\frac{2}{\pi} \int_{0}^{\infty} \frac{\sin (x t-2 n \theta) t d t}{1+t^{2}},
$$

where $t=\tan \theta$. When $2 n$ is an odd integer $k_{2 n}^{\prime}(x)$ becomes infinite as $x \rightarrow 0$ but in any case

$$
\begin{aligned}
k_{2 n}^{\prime}(\epsilon)-k_{2 n}^{\prime}(-\epsilon) & =-\frac{4}{\pi} \int_{0}^{\infty} \frac{[\sin (\epsilon t)][\cos 2 n \theta] t d t}{1+t^{2}} \\
& =-\frac{4}{\pi} \int_{0}^{\infty} \frac{[\sin u][\cos 2 n \theta] u d u}{\epsilon^{2}+u^{2}}, \tan \theta=\frac{u}{\epsilon}, \\
& \rightarrow-\frac{4}{\pi}(\cos n \pi) \int_{0}^{\infty} \sin u d u / u=-2 \cos n \pi .
\end{aligned}
$$

Hence

$$
\begin{aligned}
P \int_{-\infty}^{\infty} k_{2 n}(x) k_{2 m}(x) \frac{d x}{x} & =\frac{1}{\pi(m-n)}\left[\frac{1}{m} \sin m \pi \cos n \pi-\frac{1}{n} \sin n \pi \cos m \pi\right] \\
& =\frac{m+n}{2 \pi m n}\left[\frac{\sin (m-n) \pi}{m-n}-\frac{\sin (m+n) \pi}{m+n}\right] .
\end{aligned}
$$

This formula may be used with (4.1) to obtain the following generalization of (2.91):

$$
\int_{-\infty}^{\infty} k_{2 n}(x) k_{2 m}(x) d x=\frac{\sin [(n-m) \pi]}{\pi(m-n+1)(m-n)(m-n-1)} .
$$

When $m$ and $n$ are integers,

$$
\begin{aligned}
P \int_{-\infty}^{\infty} k_{2 n+1}(x) k_{2 m+1}(x) \frac{d x}{x} & =0, \quad m \neq n, \\
& =\frac{2}{\pi(2 n+1)}, \quad m=n .
\end{aligned}
$$

7. The interpolation formula. It is useful to have an alternative definition of $k_{n}(x)$ from which its properties may be developed. Such a definition is obtained by making use of the cardinal function of interpolation theory, the properties of which have been developed by Professor E. T. Whittaker.* We thus write for all real values of $n$ and $x$

* Proceedings of the Royal Society of Edinburgh, vol. 35 (1915), p. 181. See also W. L. Ferrar, ibid., vol. 45 (1925), p. 269, vol. 46(1926), p.323, vol. 47 (1927), p. 230; J. M. Whittaker, Proceedings of the Edinburgh Mathematical Society, (2), vol. 1 (1927), pp. 41, 169; E. T. Copson, ibid., p. 129. 


$$
k_{n}(x)=\frac{2}{\pi} \sum_{m=-\infty}^{\infty} \frac{\sin (2 m-n) \frac{\pi}{2}}{2 m-n} k_{2 m}(x) .
$$

The absolute convergence of this expansion may be established with the aid of Fejér's asymptotic formula* for $L_{n}(2 x)$. Assuming $x>0$, we may write

$$
L_{n}(2 x) \sim \pi^{-1 / 2} e^{x}(2 n x)^{-1 / 4} \cos \left[(8 n x)^{1 / 2}-\frac{\pi}{4}\right]+O\left(n^{-1 / 2}\right),
$$

and make use of the relation

$$
k_{2 m}(x)=(-1)^{m} e^{-x}\left[L_{m}(2 x)-L_{m-1}(2 x)\right] .
$$

The two Laguerre series obtained by substituting the last expression in (7.1) are also absolutely convergent and so we may add them together in a manner different from that adopted in (7.1), and obtain a single Laguerre series

$$
e^{x} k_{n}(x)=-\frac{4}{\pi} \sin \left(\frac{n \pi}{2}\right) \sum_{m=0}^{\infty} \frac{L_{m}(2 x)}{(2 m-n)(2 m+2-n)},
$$

which is absolutely and uniformly convergent for all positive values of $x$ including zero. Making use of the equation

$$
\int_{0}^{2 x} L_{m}(u) d u=L_{m}(2 x)-L_{m+1}(2 x)
$$

it is readily seen that

$$
\begin{aligned}
\int_{0}^{x} e^{u} k_{n}(u) d u & =\frac{2}{\pi} \sin \left(\frac{n \pi}{2}\right) \sum_{m=0}^{\infty} \frac{L_{m+1}(2 x)-L_{m}(2 x)}{(2 m-n)(2 m+2-n)} \\
& =\frac{1}{2} e^{x}\left[k_{n+2}(x)+k_{n}(x)\right]-\frac{2}{\pi} \sin \frac{n \pi}{2} \frac{1}{n(n+2)} .
\end{aligned}
$$

Differentiating this equation with respect to $x$ we obtain the relation

$$
k_{11}(x)-k_{n+2}(x)=k_{n+2}^{\prime}(x)+k_{n}^{\prime}(x) .
$$

Again, if we make use of the well known relation

$$
(n+1) L_{n+1}(u)-(2 n+1-u) L_{n}(u)+n L_{n-1}(u)=0,
$$

it is seen from (7.3) that $k_{n}(x)$ satisfies the difference equation

$$
(n-2) k_{n-2}(x)+(n+2) k_{n+2}(x)=(4 x-2 n) k_{n}(x),
$$

* Simple proofs of the theorem are given by Szegö, Mathematische Zeitschrift, vol. 1 (1918), p. 341, and O. Perron, Journal für die reine und angewandte Mathematik, vol. 151 (1920), p. 163. 
and with the aid of (4.3) the relation

$$
4 x k_{n}^{\prime}(x)=(n-2) k_{n-2}(x)-(n+2) k_{n+2}(x)
$$

can be established.

The differential equation (5.1) may now be obtained as in $\$ 5$ and with the aid of (7.1) we may obtain the further relations

$$
\begin{aligned}
k_{-n}(x) & =k_{n}(-x), \\
k_{n}(0) & =\frac{2}{n \pi} \sin \frac{n \pi}{2} .
\end{aligned}
$$

Thus all the principal properties of the function $k_{n}(x)$ have been obtained directly from the interpolation formula (7.1). The value of the integral

$$
\int_{0}^{\infty} k_{n}(x) k_{2 s}(x) \frac{d x}{x}
$$

may be calculated by making use of the B-test* for the integration of an in finite series over an infinite range. In using this test we make use of Szegö's inequality $\dagger$

$$
e^{-x}\left|L_{m}(2 x)\right| \leqq 1, x \geqq 0,
$$

and the inequality of $\$ 4$

to prove that the series

$$
\begin{aligned}
\left|k_{2 s}(x)\right| & <\frac{2 s}{x}, \quad x>1, \\
& <x \phi(s), \quad 0<x<1,
\end{aligned}
$$

$$
\sum_{m=0}^{\infty} \frac{1}{(2 m-n)(2 m+2-n)} \int_{0}^{\infty}\left|k_{2 s}(x)\right| e^{-x}\left|L_{m}(2 x)\right| d x
$$

converges.

The definite integral

$$
\int_{0}^{\infty} k_{2 s}(x)(d x / x) e^{-x} L_{m}(2 x)
$$

is calculated with the aid of (2.81) and is found to have the value

$$
\begin{aligned}
\frac{1}{s}, & m \geqq s ; \\
0, & m<s .
\end{aligned}
$$

* Bromwich's Infinite Series, p. 453 (1st edition).

$\dagger$ Mathematische Zeitschrift, vol. 1 (1918), p. 341. 
It is thus found that when $s$ is a positive integer

$$
\int_{0}^{\infty} k_{n}(x) k_{20}(x) \frac{d x}{x}=\frac{4}{\pi n} \frac{\sin (2 s-n) \frac{\pi}{2}}{2 s-n} .
$$

Taking (6.2) into consideration we can regard (7.1) as the $k$-series* for the function $k_{n}(x)$ whether this function is defined by (1.1) or (7.1).

The B-test may also be used to calculate the integral

$$
\int_{0}^{\infty} k_{n}(x) e^{-x} L_{m^{\prime}}(2 x) d x
$$

by the integration of (7.3) term by term. In this case we make use of (2.81) and a combination of (4.6) and (4.7) to prove the convergence of the series

$$
\sum_{m=0}^{\infty} \frac{1}{(2 m-n)(2 m+2-n)} \int_{0}^{\infty} e^{-2 x}\left|L_{m^{\prime}}(2 x)\right|\left|L_{m}(2 x)\right| d x .
$$

In this way it may be shown that (7.3) is the Laguerre series for the function $k_{n}(x) e^{x}$ whichever definition is adopted for $k_{n}(x)$.

When $k_{n}(x)$ is defined with the aid of (7.3) the integral (6.5) may be calculated by using the Parseval theorem for the Laguerre functions. $\dagger$ The analysis leads to the interesting equation

(7.7) $\cot (m \pi)-\cot (n \pi)=\frac{1}{2 \pi} \sum_{s=-\infty}^{\infty} \frac{(n-m)(m-n+1)(n-m+1)}{(s-n)(s-m)(s+1-n)(s+1-m)}$,

which holds for both real and complex values of $m$ and $n$, as may be seen by comparing the residues of the functions of $m$ on the two sides of the equation.

The equivalence of the two definitions of $k_{n}(x)$ may be inferred from the fact that the two functions have the same Laguerre series or it may be proved by means of Parseval's theorem for Fourier series, the two functions $f(\theta)$, $g(\theta)$ in the integral

$$
\frac{1}{\pi} \int_{-\pi}^{\pi} f(\theta) g(\theta) d \theta
$$

being defined as follows:

* The $k$-series for a function $f(x)$ is of type $f(0) k_{0}(x)+c_{1} k_{2}(x)+\cdots$ where the coefficients $c_{n}$ are calculated with the aid of the orthogonal relation of $\$ 6$.

$\dagger$ For this theorem see S. Wigert, Arkiv för Matematik, Astronomi och Fysik, vol. 15 (1921); M. Riesz, Acta Litterarum ac Scientiarum regiae Universitatis Hungaricae Francisco-Josephinae, vol. 1 (1923), p. 209.

$\ddagger$ It should be noticed that $f(\theta)$ is not of bounded variation in the interval $(-\pi, \pi)$ and is discontinuous at the points $\theta= \pm \pi / 2$. The convergence of its Fourier series is discussed briefly in $\$ 9$. A really elementary proof of the equivalence of the two definitions of $k_{n}(x)$ has not been obtained. 


$$
\begin{array}{ccc}
f(\theta)=e^{i x \tan \theta}, & -\pi<\theta<\pi ; \\
g(\theta)=2 e^{-i n \theta}, & -\pi<2 \theta<\pi, \\
=0, & -2 \pi<2 \theta<-\pi, \\
\quad \text { or } & \pi<2 \theta<2 \pi ;
\end{array}
$$

the integral then becomes equal to the integral (1.1) defining $k_{n}(x)$ while the Parseval series becomes identical with (7.1). The use of an interpolation formula of type (7.1) for the representation of a function of $n$ in terms of its values when $n$ is an integer (or has even integral values) is not new. W. L. Ferrar has kindly informed me that the Legendre function $P_{n}(x)$ was expressed as a series of Legendre polynomials by J. Dougall* long ago. The interpolation formula was also used by de la Vallée Poussin $\dagger$ to approximate to the value of a function over a limited range.

8. The exponential integral. If $a$ is positive and $n$ is a positive integer,

$$
\begin{aligned}
\int_{0}^{\infty} e^{-a x} k_{2 n}(x) d x & =\frac{(-1)^{n}}{n !} \int_{0}^{\infty} x e^{x(1-a)} \frac{d^{n}}{d x^{n}}\left[e^{-2 x} x^{n-1}\right] d x \\
& =\frac{1}{n !} \int_{0}^{\infty} e^{-2 x} x^{n-1} \frac{d^{n}}{d x^{n}}\left\{x e^{x(1-a)}\right\} d x \\
& =\frac{1}{n !} \int_{0}^{\infty} e^{-x(1+a)}\left\{x^{n}(1-a)^{n}+n x^{n-1}(1-a)^{n-1}\right\} d x \\
& =\frac{(1-a)^{n}}{(1+a)^{n+1}}+\frac{(1-a)^{n-1}}{(1+a)^{n}}=\frac{2}{(1+a)^{2}}\left(\frac{1-a}{1+a}\right)^{n-1} .
\end{aligned}
$$

On the other hand,

$$
\int_{0}^{\infty} e^{-a x} k_{0}(x) d x=\frac{1}{1+a} .
$$

A general formula valid for all real values of $n$ may be obtained from (7.3) with the aid of Parseval's theorem for the Laguerre functions and the known formula

$$
\int_{0}^{\infty} e^{-x(1+a)} L_{m}(2 x) d x=(-1)^{m} \frac{(1-a)^{m}}{(1+a)^{m+1}},
$$

which gives the Laguerre constants for the function $e^{-x(a-1)}$. The formula may be written in the two forms

* Proceedings of the Edinburgh Mathematical Society, vol. 18 (1900), p. 79. See also H. B. C. Darling, Quarterly Journal of Mathematics, vol. 49 (1923), p. 289.

† Bulletin de l'Académie Royale de Belgique (Classe de Sciences), 1908, p. 341. See also J. M. Whittaker, Proceedings of the Edinburgh Mathematical Society, (2), vol. 1 (1928), p. 169. 


$$
\begin{aligned}
\int_{0}^{\infty} e^{-a x} k_{2 n}(x) d x= & \frac{1}{\pi} \sin n \pi \sum_{m=0}^{\infty}(-1)^{m+1} \frac{1}{(m-n)(m+1-n)} \frac{(1-a)^{m}}{(1+a)^{m+1}} \\
= & \frac{1}{n \pi} \sin n \pi\left[\frac{1}{1+a}-\frac{2 n}{(1+a)^{2}}\left\{\frac{1}{n-1}-\frac{1-a}{1+a} \frac{1}{n-2}\right.\right. \\
& \left.\left.+\left(\frac{1-a}{1+a}\right)^{2} \frac{1}{n-3}-\cdots\right\}\right]
\end{aligned}
$$

When $n$ is a positive integer the limiting form of the right hand side must be taken. When $n$ is a negative integer the integral is zero as we should expect. If

$$
y_{n}(a)=\int_{0}^{\infty} e^{-a x} k_{2 n}(x) d x,
$$

it is readily seen that $y_{n}(a)$ satisfies the difference equation

$$
y_{n+1}(a)-\frac{1-a}{1+a} y_{n}(a)=\frac{\sin n \pi}{\pi} \frac{1}{n(n+1)(1+a)} .
$$

This may be seen directly with the aid of (4.3).

9. Some special series. The relation (2.7) and Fejér's asymptotic formula (7.2) may be used to prove that when $m$ is a positive integer $k_{2 m}(x) \rightarrow 0$ as $m \rightarrow \infty$ and the same result may be derived from the following asymptotic formula which is derived from a result given by Perron (loc. cit.):

$$
\pi^{1 / 2} k_{2 m}(x) \sim(-1)^{m+1}(2 x)(2 m x)^{-3 / 4} \cos \left[(8 m x)^{1 / 2}-3 \pi / 4\right] .
$$

Since $k_{2 m}(x) \rightarrow 0$ it follows from Fatou's theorem* that the power series (2.2) converges at all regular points on the circle of convergence. The point $s=-1$ is the only irregular point and from the extension of Fatou's theorem given by Riesz $\dagger$ it may be concluded that the convergence is uniform on any arc which does not contain the point $s=-1$. The same result can be derived also from the theory of Fourier series. $\ddagger$

To examine the convergence of the series at the point $s=-1$ we first note that the relation (4.3) gives

$$
k_{0}^{\prime}(x)+2 k_{2}^{\prime}(x)+2 k_{4}^{\prime}(x)+\cdots+2 k_{2 n-2}^{\prime}(x)+k_{2 n}^{\prime}(x)=k_{0}(x)-k_{2 n}(x) .
$$

* P. Fatou, Acta Mathematica, vol. 30 (1906), p. 335.

$\dagger$ M. Riesz, Journal für die reine und angewandte Mathematik, vol. 140 (1911), p. 89.

$\ddagger$ A similar result for the series defining the generalised Laguerre polynomials is mentioned by E. Hille, Proceedings of the National Academy of Sciences, vol. 12 (1926), p. 261. See also G. Szegö, Mathematische Zeitschrift, vol. 25 (1926), p. 87. 
The asymptotic formulas indicate that if $0 \leqq x \leqq a$ we can find a number $m$ independent of $x$ such that for $n>m$

$$
\left|k_{2 n}(x)\right|<\epsilon
$$

where $\epsilon$ is any preassigned small positive quantity. The series on the left can be regarded, then, as converging uniformly in $x$ when we put $n=\infty$, and since $k_{0}(x)=e^{-x}, k_{0}^{\prime}(x)=-e^{-x}$ it seems that the series converges uniformly to zero. Integrating it term by term we find that, when $0 \leqq x \leqq a$,

$$
k_{0}(x)+k_{2}(x)+\cdots+k_{2 n}(x) \rightarrow 1 \text { uniformly as } n \rightarrow \infty .
$$

Again, the relation (4.3) gives

$$
\begin{aligned}
k_{0}(x)-k_{2}(x)+k_{4}(x)-\cdots+ & k_{4 n}(x)-k_{4 n+2}(x) \\
& =k_{0}^{\prime}(x)+k_{2}^{\prime}(x)+k_{4}^{\prime}(x)+\cdots+k_{4 n+2}^{\prime}(x) .
\end{aligned}
$$

As $n \rightarrow \infty$ the series on the right tends uniformly to zero hence the series on the left also tends uniformly to zero. This establishes the convergence of the power series (2.2) for $s=-1$ and of the series (2.1) for $\alpha=0, \theta=\pi / 2$.

Putting $\alpha=0, \theta=\pi / 4$, we find that

$$
\begin{aligned}
& \cos x=k_{0}(x)-k_{4}(x)+k_{8}(x)-\cdots, \\
& \sin x=k_{2}(x)-k_{6}(x)+k_{10}(x)-\cdots,
\end{aligned}
$$

and in general, if $2 n \theta=\pi$,

$$
e^{i x \tan \theta}=N_{0}(x)+e^{2 i \theta} N_{2}(x)+\cdots+e^{2(n-1) i \theta} N_{2(n-1)}(x),
$$

where

$$
\begin{aligned}
& N_{0}(x)=k_{0}(x)-k_{2 n}(x)+k_{4 n}(x)-\cdots, \\
& N_{2}(x)=k_{2}(x)-k_{2 n+2}(x)+k_{4 n+2}(x)-\cdots,
\end{aligned}
$$

Furthermore, with this value of $\theta$,

$$
\begin{gathered}
e^{i x \tan 3 \theta}=N_{0}(x)+e^{6 i \theta} N_{2}(x)+\cdots+e^{6(n-1) i \theta} N_{2(n-1)}(x), \\
e^{i x \tan 5 \theta}=N_{0}(x)+e^{10 i \theta} N_{2}(x)+\cdots .
\end{gathered}
$$

It can, indeed, be shown directly from (2.3) that, if $D \equiv d / d x$,

$$
\begin{aligned}
& N_{0}(x)=D\left[N_{2}(x)+N_{4}(x)+\cdots+N_{2(n-1)}(x)\right], \\
& D N_{0}(x)+N_{2}(x)=D\left[N_{4}(x)+N_{6}(x)+\cdots+N_{2(n-1)}(x)\right] \text {, } \\
& D\left[N_{0}(x)+N_{2}(x)+\cdots+N_{2 n-4}(x)\right]+N_{2(n-1)}(x)=0 .
\end{aligned}
$$


If

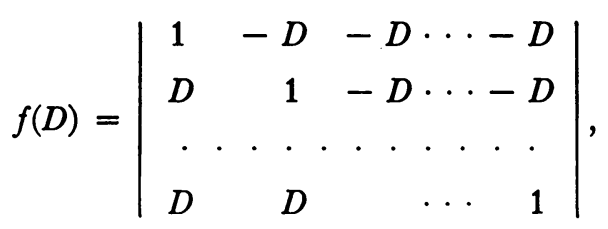

where the determinant has $n$ rows and $n$ columns, the differential equation $f(D) w=0$ has the $n$ particular solutions

$$
w_{1}=e^{i x \tan \theta}, w_{2}=e^{i x \tan 3 \theta}, \cdots, w_{n}=e^{i x \tan (2 n-1) \theta} .
$$

To see this we consider the set of $n$ linear equations

$$
\begin{aligned}
& x_{0}=i \tan \theta\left[x_{1}+x_{2}+\cdots+x_{n-1}\right], \\
& i x_{0} \tan \theta+x_{1}=i \tan \theta\left[x_{2}+x_{3}+\cdots+x_{n-1}\right] \text {, } \\
& i\left(x_{0}+x_{1}+\cdots+x_{n-2}\right) \tan \theta+x_{n-1}=0 \text {. }
\end{aligned}
$$

Writing $\lambda=x_{0}+x_{1}+x_{2}+\cdots+x_{n-1}$, we find successively

$$
\begin{aligned}
x_{0} & =i \lambda \sin \theta e^{-i \theta}, \quad x_{1}=i \lambda \sin \theta e^{-3 i \theta}, \\
x_{2} & =i \lambda \sin \theta e^{-5 i \theta}, \cdots, x_{n-1}=i \lambda \sin \theta e^{-(2 n-1) i \theta}, \\
\lambda & =i \lambda \sin \theta e^{-i \theta}\left[1+e^{-2 i \theta}+\cdots+e^{-(2 n-2) i \theta}\right] \\
& =i \lambda \sin \theta e^{-i \theta} \frac{1-e^{-2 n i \theta}}{1-e^{-2 i \theta}}=\frac{\lambda}{2}\left[1-e^{-2 n i \theta}\right] .
\end{aligned}
$$

Hence, if $\lambda \neq 0$ we must have $e^{2 n i \theta}=-1$ or $2 n \theta=s \pi$, where $s$ is an odd integer. It is easily seen that

$$
\begin{aligned}
& n N_{0}(x)=w_{1}+w_{2}+\cdots+w_{n}, \\
& n N_{2}(x)=w_{1} e^{-2 i \theta}+w_{2} e^{-6 i \theta}+\cdots+w_{n} e^{-2 i(2 n-1)} \theta, \\
& n N_{4}(x)=w_{1} e^{-4 i \theta}+w_{2} e^{-12 i \theta}+\cdots+w_{n} e^{-4 i(2 n-1) i \theta},
\end{aligned}
$$

and so on.

California Institute of Technology,

Pasadena, Calif. 